\title{
Caractéristiques et issues des hospitalisations pour les cas de COVID-19 et d'influenza dans la région de Toronto
}

Amol A. Verma MD MPh, Tejasvi Hora BSc, Hae Young Jung MSc, Michael Fralick MD PhD, Sarah L. Malecki MD MSc, Lauren Lapointe-Shaw MD PhD, Adina Weinerman MD MScS, Terence Tang MD, Janice L. Kwan MD MSP, Jessica J. Liu MD, Shail Rawal MD MSP, Timothy C.Y. Chan PhD LEL, Angela M. Cheung MD PhD, Laura C. Rosella PhD, Marzyeh Ghassemi PhD, Margaret Herridge MD MSc, Muhammad Mamdani MSP PharmD, Fahad Razak MD MSc

- Citation : CMAJ 2021 March 22;193:E410-8. doi : 10.1503/cmaj.202795-f; diffusion hâtive le 10 février 2021

Voir la version anglaise de l'article ici : www.cmaj.ca/lookup/doi/10.1503/cmaj.202795

Infographie accessible en anglais au : www.cmaj.ca/lookup/doi/10.1503/cmaj.202795/tab-related-content

\section{RÉSUMÉ}

CONTEXTE : Les caractéristiques des patients, les soins cliniques, l'utilisation des ressources et les issues cliniques des personnes atteintes de la maladie à coronavirus 2019 (COVID-19) hospitalisées au Canada ne sont pas bien connus.

MÉTHODES : Nous avons recueilli des données sur tous les adultes hospitalisés atteints de la COVID-19 ou de l'influenza ayant obtenu leur congé d'unités médicales ou d'unités de soins intensifs médicaux et chirurgicaux entre le $1^{\text {er }}$ novembre 2019 et le 30 juin 2020 dans 7 centres hospitaliers de Toronto et de Mississauga (Ontario). Nous avons comparé les issues cliniques des patients à l'aide de modèles de régression multivariée, en tenant compte des facteurs sociodémographiques et de l'intensité des comorbidités. Nous avons validé le degré d'exactitude de 7 scores de risque mis au point à l'externe pour déterminer leur capacité à prédire le risque de décès chez les patients atteints de la COVID-19.
RÉSULTATS : Parmi les hospitalisations retenues, 1027 patients étaient atteints de la COVID-19 (âge médian de 65 ans, 59,1\% d'hommes) et 783 étaient atteints de l'influenza (âge médian de 68 ans, 50,8\% d'hommes). Les patients âgés de moins de 50 ans comptaient pour $21,2 \%$ de toutes les hospitalisations dues à la COVID-19 et $24,0 \%$ des séjours aux soins intensifs. Comparativement aux patients atteints de l'influenza, les patients atteints de la COVID-19 présentaient un taux de mortalité perhospitalière (mortalité non ajustée $19,9 \%$ c. $6,1 \%$; risque relatif [RR] ajusté $3,46 \%$, intervalle de confiance [IC] à $95 \% 2,56-4,68$ ) et un taux d'utilisation des ressources des unités de soins intensifs (taux non ajusté $26,4 \%$ c. 18,0\%; RR ajusté 1,50 , IC à $95 \% 1,25-1,80$ ) significativement plus élevés, ainsi qu'une durée d'hospitalisation (durée médiane non ajustée 8,7 jours c. 4,8 jours; rapport des taux d'incidence ajusté 1,45 ; IC à $95 \% 1,25-1,69)$ significativement plus longue. Le taux de réhospitalisation dans les 30 jours n'était pas significativement différent (taux non ajusté $9,3 \%$ c. 9,6\%; RR ajusté $0,98 \%$, IC à $95 \% 0,70-1,39)$. Trois scores de risque utilisant un pointage pour prédire la mortalité perhospitalière ont montré une bonne discrimination (aire sous la courbe $[\mathrm{ASC}]$ de la fonction d'efficacité du récepteur [ROC] 0,72-0,81) et une bonne calibration.

INTERPRÉTATION : Durant la première vague de la pandémie, l'hospitalisation des patients atteints de la COVID-19 était associée à des taux de mortalité et d'utilisation des ressources des unités de soins intensifs et à une durée d'hospitalisation significativement plus importants que les hospitalisations des patients atteints de l'influenza. De simples scores de risque peuvent prédire avec une bonne exactitude le risque de mortalité perhospitalière des patients atteints de la COVID-19. 
D es études internationales ont rapporté que les patients hospitalisés en raison de la maladie à coronavirus 2019 (COVID-19) présentaient des taux élevés de maladie grave et de mortalitén ${ }^{-5}$. Deux petites études de cas canadiennes ont décrit les soins donnés aux patients atteints d'une forme grave de la COVID-19 et ont fait état de taux de mortalité pouvant atteindre $25 \% \%^{6,7}$. Cependant, on en sait peu sur les issues cliniques des patients hospitalisés en raison de la COVID-19 au Canada, particulièrement à l'extérieur des unités de soins intensifs (USI). Le taux de létalité de la COVID-19 varie grandement selon le pays ${ }^{8}$, et les issues des patients hospitalisés au Canada pour la COVID-19 peuvent être différentes de celles des patients d'autres pays en raison des différences démographiques, des mesures de santé publique et des systèmes de santé.

La grippe saisonnière est un bon outil de comparaison pour la COVID-199-11, puisqu'elle est causée par un autre virus respiratoire, qu'elle est bien répandue dans la population et qu'elle est associée à des taux de morbidité et de mortalité élevés. Le but de la présente étude était de décrire les caractéristiques des patients, l'utilisation des ressources, les soins cliniques et les issues dans une cohorte de patients hospitalisés en raison de la COVID-19 en Ontario (Canada), et de les comparer à ceux de patients hospitalisés en raison de l'influenza. Nous avons aussi validé la capacité de différents scores de risque pronostiques à prédire avec exactitude la mortalité perhospitalière chez les patients atteints de la COVID-19.

\section{Méthodes}

\section{Conception et conditions de l'étude}

Nous avons réalisé une étude de cohorte rétrospective à partir des données de 7 grands centres hospitaliers ( 5 centres universitaires et 2 hôpitaux communautaires d'enseignement) de Toronto et de Mississauga (Ontario) participant au projet de recherche collaborative en milieu hospitalier GEMIN ${ }^{12}$.

\section{Collecte des données}

Nous avons recueilli des données administratives et cliniques à partir des systèmes d'information hospitaliers du projet GEMINI, comme décrit précédemment ${ }^{12,13}$. Nous avons colligé des données sur les caractéristiques démographiques des patients, l'utilisation des ressources hospitalières et les issues cliniques indiquées par l'hôpital à partir de la Base de données sur les congés des patients et le Système national d'information sur les soins ambulatoires de l'Institut canadien d'information sur la santé (ICIS). Nous avons également extrait directement des systèmes d'information hospitaliers des données cliniques additionnelles : résultats de tests de laboratoire et d'examens radiologiques, signes vitaux et prescriptions de médicaments durant l'hospitalisation (consulter l'annexe 1, accessible en anglais au www.cmaj.ca/lookup/ doi/10.1503/cmaj.202795/tab-related-content pour de plus amples détails concernant la qualité des données).

\section{Population étudiée}

Nous avons inclus tous les adultes de plus de 18 ans hospitalisés dans des unités médicales ou des unités de soins intensifs médi- caux et chirurgicaux, y compris des unités de soins coronariens, et qui ont obtenu leur congé entre le $1^{\text {er }}$ novembre 2019 et le 30 juin 2020. Les unités médicales comprennent toutes les spécialités médicales (médecine générale, cardiologie, pneumologie, etc.). Les données incluent tous les cas d'hospitalisations pour la COVID-19 et l'influenza ainsi que leurs complications médicales, mais elles pourraient ne pas tenir compte d'un petit nombre de patients atteints de la COVID-19 ou de l'influenza admis pour des raisons non médicales et n'ayant pas été transférés à un service médical par la suite.

Ont été considérés comme atteints de la COVID-19 les patients répondant aux critères de la version canadienne améliorée des codes de la Classification internationale des maladies et des problèmes de santé connexes, $10^{e}$ révision (CIM-10-CA), nommément U07.1 (« des résultats de laboratoire confirment le diagnostic de COVID-1914 ») et U07.2 (« un diagnostic de COVID19 est posé à partir de la documentation clinique ou épidémiologique, mais les résultats de laboratoire ne sont pas concluants, ne sont pas disponibles ou aucun dépistage n'a été réalisé $\left.{ }^{14} »\right)$. Dans 150 centres hospitaliers aux États-Unis, le code U07.1 était sensible à $98 \%$ et spécifique à $99 \%$ pour la COVID-19 15 . Les patients atteints de l'influenza ont été identifiés selon un algorithme validé basé sur la classification CIM-10-CA (codes J09, J10.0, J10.1, J10.8, J11.0, J11.1 et J11.8) qui était sensible à $83 \%$ et spécifique a $98 \%$ pour l'influenza en Ontario ${ }^{16}$. Moins de 6 patients étaient atteints simultanément de la COVID-19 et de l'influenza (le nombre exact a été supprimé pour limiter le risque de comptage en double); ils ont été intégrés au groupe COVID-19.

\section{Issues cliniques et mesure}

Les 5 principales issues cliniques à l'étude étaient la mortalité perhospitalière, la réadmission non planifiée dans une unité médicale ou une unité de soins intensifs médicaux ou chirurgicaux de l'un des centres hospitaliers participants dans les 30 jours suivant le congé, l'hospitalisation à l'unité de soins intensifs, la durée totale du séjour hospitalier et la durée du séjour à l'unité de soins intensifs. Nous avons aussi mesuré le taux de réadmission dans les 7 jours suivant le congé et la durée du séjour au service des urgences.

En outre, nous avons analysé l'utilisation de la tomodensitométrie (TDM) thoracique en raison de son rôle dans le diagnostic de la COVID-1917, ainsi que la prescription chez les patients hospitalisés d'antibiotiques fréquemment utilisés pour traiter des infections pulmonaires (voir l'annexe 1) 18-20 $^{18}$ d'anticoagulants et de corticostéroïdes systémiques. La prescription de ces médicaments pourrait avoir été associée à la COVID-1921,22; il faut toutefois noter que la période à l'étude était antérieure à la publication des résultats de l'essai clinique RECOVERY portant sur la dexaméthasone ${ }^{21}$. Nous avons utilisé les codes de la Classification canadienne des interventions en santé, élaborée par l'ICIS, pour recenser le recours à la ventilation artificielle effractive, à la dialyse (dialyse nouvellement amorcée ou dialyse chronique), à l'endoscopie gastro-intestinale et à la bronchoscopie. La dialyse pourrait être plus fréquemment requise chez les patients atteints de la COVID-1923, et l'endoscopie gastro-intestinale et la 
bronchoscopie sont des procédures effractives fréquentes dont le potentiel de contribution à la transmission de la COVID-19 est source de préoccupations ${ }^{24,25}$.

\section{Caractéristiques des patients}

Pour chaque patient, nous rapportons l'âge et le sexe, la résidence dans un établissement de soins de longue durée et le transfert depuis un établissement de soins de courte durée, de même que les résultats de tests de laboratoire et les signes vitaux à l'arrivée. Nous avons catégorisé les comorbidités selon les codes de la CIM-10-CA à l'aide du logiciel Clinical Classification Software Refined ${ }^{26,27}$ et l'indice de comorbidité de Charlson ${ }^{28}$. Puisque le revenu et l'origine ethnique des patients n'étaient pas disponibles, nous avons utilisé le code postal pour rendre compte du revenu à l'échelle du quartier et de la proportion de la population qui s'identifie comme appartenant à une minorité visible (voir l'annexe 1$)^{29,30}$. Les quartiers ont été classés en quintiles (Q1 à Q5), soit du revenu le plus faible au plus élevé, et selon la proportion de la population qui s'identifie comme appartenant à une minorité visible, respectivement.

\section{Scores prédictifs de la mortalité}

Nous avons calculé le risque de mortalité perhospitalière chez les patients atteints de la COVID-19 et de l'influenza à partir d'une adaptation de 7 scores, en fonction des données démographiques et cliniques obtenues dans les 24 premières heures après l'admission. Le score Acute Physiology and Chronic Health Evaluation modifié (mAPACHE) ${ }^{31}$ et le système de classification des maladies graves (CISSS) ${ }^{32}$ ont été conçus pour prédire le risque de mortalité à l'unité de soins intensifs et de mortalité dans les 30 jours à l'aide de données cliniques numériques systématiquement recueillies. Nous avons aussi sélectionné les 4 modèles $\left(\mathrm{Lu}^{33} \mathrm{Hu},{ }^{34} \mathrm{Xie}^{35}\right.$ et NEWS2 ${ }^{36}$ ) ayant le mieux performé dans une étude de validation externe ${ }^{37}$ du Royaume-Uni portant sur des modèles recensés dans une revue systématique de modèles de prédiction de la mortalité due à la COVID-1938. Enfin, nous avons inclus le score de mortalité ISARIC Coronavirus Clinical Characterisation Consortium 4C (ISARIC-4C) ${ }^{39}$, lequel a été dérivé d'une large cohorte du Royaume-Uni (voir l'annexe 1 pour de plus amples détails).

\section{Analyse statistique}

Nous avons comparé les caractéristiques de base des patients atteints de la COVID-19 et de ceux atteints de l'influenza à l'aide de différences normalisées, les différences supérieures à 0,1 étant évocatrices d'un déséquilibre entre les groupes ${ }^{40}$. Nous avons comparé les différences non ajustées dans les soins cliniques, l'utilisation des ressources et les issues cliniques à l'aide du test de $\chi^{2}$, du test $t$ de Student et du test de Mann-Whitney pour les variables catégorielles, les variables continues à distribution symétrique et les variables continues ne suivant pas une distribution normale, respectivement. Afin de tenir compte des comparaisons multiples, nous avons utilisé la correction de Bonferroni pour corriger les valeurs $p$ pour l'ensemble des comparaisons, sauf dans le cas des 5 principales issues à l'étude.
Nous avons utilisé la régression multivariée pour comparer les issues après avoir procédé à un ajustement selon l'âge du patient, le sexe, le score de Charlson, la résidence en établissement de soins de longue durée, le revenu moyen et la proportion de la population qui s'identifie comme appartenant à une minorité visible dans son quartier ainsi que le centre hospitalier où il a été admis. Les covariables ont été sélectionnées a priori en fonction d'associations avec la mortalité rapportées précédemment dans le contexte de la COVID-1910,41-43. Nous avons employé une régression de Poisson ${ }^{44}$ pour modéliser le risque de mortalité, de réhospitalisation et d'hospitalisation à l'unité de soins intensifs afin de générer des risques relatifs et d'éviter l'interprétation erronée des rapports de cotes obtenus par régression logistique. Une régression binomiale négative a été appliquée pour modéliser la durée de l'hospitalisation et la durée du séjour à l'unité de soins intensifs. Pour présenter la capacité des scores de risque à prédire avec exactitude le risque de mortalité perhospitalière dans le contexte de la COVID-19, nous avons calculé l'aire sous la courbe (ASC) de la fonction d'efficacité du récepteur ainsi que la sensibilité, la spécificité, la valeur prédictive négative et la valeur prédictive positive à différents seuils. La représentation visuelle du calage du modèle compare les scores modélisés aux proportions des issues observées pour les systèmes à pointage, et des courbes de calage ajustées par régression locale (Loess) comparent les probabilités observées aux probabilités prévues, pour les systèmes basés sur des scores de probabilité.

\section{Analyses des sous-groupes et sensibilité}

Tout d'abord, nous avons examiné les issues stratifiées selon le groupe d'âge, en utilisant a priori les groupes $<50$ ans, 50-75 ans et $>75$ ans. Deuxièmement, nous avons décrit les caractéristiques et les issues des patients hospitalisés à l'unité des soins intensifs. Troisièmement, afin de déterminer si les décès étaient dus, plutôt qu'associés, à la COVID-19, nous rapportons le diagnostic "le plus responsable » (principal) au moment du congé chez les patients atteints de la COVID-19 hospitalisés. Quatrièmement, nous avons refait toutes les analyses, cette fois en excluant les patients à qui on avait attribué le code U07.2 (absence de confirmation d'un diagnostic de COVID-19 par un test en laboratoire). Cinquièmement, puisque les centres hospitaliers participants étaient des centres de soins tertiaires et quaternaires dotés de grandes unités de soins intensifs, nous avons répété les analyses en incluant uniquement les patients hospitalisés depuis le service des urgences, ce qui exclut les transferts entre établissements, qui pourraient mener à une surestimation de la gravité de la maladie étant donné qu'ils concernent principalement des patients transférés aux soins intensifs. Sixièmement, afin de tenir compte des facteurs de risque concurrents, nous avons considéré dans notre modèle l'hospitalisation à l'unité des soins intensifs et le décès comme une issue mixte. Enfin, pour tenir compte de l'accumulation de données pour certains patients, nous avons refait nos analyses principales en choisissant au hasard une seule hospitalisation pour représenter les patients aux hospitalisations multiples. 


\section{Approbation éthique}

Nous avons obtenu l'approbation des comités d'éthique de la recherche du Réseau universitaire de santé (Toronto), du Centre des sciences de la santé Sunnybrook (Toronto) et de l'Hôpital St. Michael (Toronto) par la plateforme intégrée de Clinical Trials Ontario; l'Hôpital St. Michael a agi à titre de comité d'éthique de référence. Les comités d'éthique de la recherche de Trillium Health Partners (Mississauga) et de l'Hôpital Mount Sinai (Toronto) ont aussi donné leur approbation.

\section{Résultats}

La cohorte comprenait 783 patients hospitalisés en raison de l'influenza (763 patients distincts) et 1027 patients hospitalisés en raison de la COVID-19, dont 944 ayant un diagnostic confirmé par un test en laboratoire (972 patients distincts). Cela représentait $23,5 \%$ de toutes les hospitalisations pour la COVID-19 en Ontario $(n=4373)^{45}$ durant la période à l'étude.

\section{Caractéristiques des patients}

Les patients atteints de la COVID-19 et de l'influenza avaient un âge médian de 65 ans (écart interquartile [EI] : 53-79) et de 68 ans (EI : 55-80), respectivement (tableau 1). Les patients atteints de la COVID-19 étaient plus souvent des hommes (59,1\% contre $50,8 \%$ ), et étaient plus susceptibles de présenter un score de Charlson de 0 (54,1\% contre $38,8 \%$ ) et de résider dans un établissement de soins de longue durée $(11,7 \%$ contre $4,5 \%)$. Les patients qui habitaient dans des quartiers à plus faible revenu semblaient surreprésentés dans le groupe de la COVID-19 (Q1 34,2\%; Q5 10,9\%) et dans celui de l'influenza (Q1 31,7\%; Q5 12,1\%), alors qu'aucun gradient clair n'a été observé en ce qui a trait à la proportion de la population qui s'identifie comme appartenant à une minorité visible. L'hypertension et le diabète étaient des comorbidités fréquentes dans les groupes de la COVID-19 et de l'influenza, tandis que la maladie pulmonaire obstructive chronique et l'insuffisance cardiaque étaient plus fréquentes dans le groupe de l'influenza que dans celui de la COVID-19 (tableau 1).

Les différences dans les signes vitaux n'étaient pas significatives entre le groupe de la COVID-19 et celui de l'influenza. Dans les 72 premières heures d'hospitalisation, $44,9 \%$ des patients hospitalisés pour la COVID-19 ont eu besoin d'oxygénothérapie, contre $37,7 \%$ de ceux hospitalisés pour l'influenza. Les patients atteints de la COVID-19 présentaient des taux de marqueurs inflammatoires légèrement supérieurs à ceux des patients atteints de l'influenza (p. ex., protéine C-réactive, D-dimères [fragments issus de la dégradation de la fibrine], ferritine, lactate déshydrogénase); des analyses pour ces marqueurs ont été prescrites plus fréquemment chez les patients atteints de la COVID-19 que chez les autres (tableau 2).

\section{Mortalité et réhospitalisation}

Les patients atteints de la COVID-19 présentaient un taux de mortalité perhospitalière ajusté et non ajusté significativement plus élevé que ceux atteints de l'influenza (taux de
Tableau 1 : Caractéristiques des hospitalisations pour la

COVID-19 et l'influenza

\begin{tabular}{|c|c|c|}
\hline \multirow[b]{2}{*}{ Variable } & \multicolumn{2}{|c|}{$N^{\text {bre }}(\%)$ d'hospitalisations* } \\
\hline & $\begin{array}{c}\text { COVID-19 } \\
n=1027\end{array}$ & $\begin{array}{c}\text { Influenza } \\
n=783\end{array}$ \\
\hline Patients distincts & $972(94,6)$ & $763(97,4)$ \\
\hline Âge médian, années (EI) & $65(53-79)$ & $68(55-80)$ \\
\hline Groupe d'âge, années & & \\
\hline$<50$ & $218(21,2)$ & $141(18,0)$ \\
\hline $50-75$ & $480(46,7)$ & $390(49,8)$ \\
\hline$>75$ & $329(32,0)$ & $252(32,2)$ \\
\hline
\end{tabular}

\section{Sexe, hommes}

$607(59,1)$

$398(50,8)$

0,17

Score de Charlson

0

1

$556(54,1)$

$304(38,8)$

$183(17,8)$

$175(22,3)$

$\geq 2$

$288(28,0)$

$304(38,8)$

Quintile de revenu des quartiers

$\begin{array}{lcc}1 \text { (inférieur) } & 351(34,2) & 248(31,7) \\ 2 & 177(17,2) & 139(17,8) \\ 3 & 153(14,9) & 127(16,2) \\ 4 & 163(15,9) & 142(18,1) \\ 5 \text { (supérieur) } & 112(10,9) & 95(12,1) \\ \text { Manquant } & 71(6,9) & 32(4,1)\end{array}$

Quintile d'appartenance à une minorité visible dans les quartiers

\begin{tabular}{|c|c|c|c|}
\hline 1 (inférieur) & $100(9,7)$ & $100(12,8)$ & \\
\hline 2 & $196(19,1)$ & $167(21,3)$ & \\
\hline 3 & $264(25,7)$ & $171(21,8)$ & \\
\hline 4 & $181(17,6)$ & $166(21,2)$ & \\
\hline 5 (supérieur) & $212(20,6)$ & $138(17,6)$ & \\
\hline Manquant & $74(7,2)$ & $41(5,2)$ & \\
\hline $\begin{array}{l}\text { Résident d'un établissement } \\
\text { de soins de longue durée }\end{array}$ & $120(11,7)$ & $35(4,5)$ & 0,27 \\
\hline $\begin{array}{l}\text { Transfert depuis d'un } \\
\text { établissement de soins de } \\
\text { courte durée }\end{array}$ & $90(8,8)$ & $24(3,1)$ & 0,24 \\
\hline \multicolumn{4}{|l|}{ Comorbidités $\ddagger$} \\
\hline Hypertension & $356(34,7)$ & $252(32,2)$ & 0,05 \\
\hline Diabète & $284(27,7)$ & $229(29,2)$ & 0,04 \\
\hline Insuffisance rénale & $212(20,6)$ & $169(21,6)$ & 0,02 \\
\hline Troubles neurocognitifs & $174(16,9)$ & $105(13,4)$ & 0,10 \\
\hline Coronaropathie & $63(6,1)$ & $63(8,0)$ & 0,08 \\
\hline Insuffisance cardiaque & $62(6,0)$ & $98(12,5)$ & 0,23 \\
\hline MPOC & $55(5,4)$ & $96(12,3)$ & 0,25 \\
\hline
\end{tabular}

Remarque : COVID-19 = maladie à coronavirus 2019, DN = différence normalisée, EI = écart interquartile, $\mathrm{MPOC}=$ maladie pulmonaire obstructive chronique, S.O. = sans objet. ${ }^{*}$ Sauf indication contraire.

†Une DN supérieure à 0,1 indique un déséquilibre entre les groupes ${ }^{40}$.

łLes comorbidités ont été classées selon les codes de diagnostic de la CIM-10-CA à

l'aide du logiciel Clinical Classification Software Refined ${ }^{26}$. 
mortalité non ajusté $19,9 \%$ c. $6,1 \%, p<0,001$; risque relatif [RR] ajusté 3,46, intervalle de confiance [IC] à $95 \% 2,56-4,68$ ) (tableau 3, tableau 4). Chez les patients atteints de la COVID$19,4,3 \%$ et $9,3 \%$ des patients ont été réhospitalisés dans les 7 jours et dans les 30 jours suivants, respectivement, ce qui n'était pas significativement différent des patients atteints de l'influenza, avant ou après ajustement.

Parmi les patients atteints de la COVID-19 hospitalisés, les diagnostics principaux au moment du congé étaient la COVID-19, une pneumonie virale, le sepsis ou des soins palliatifs pour 183 $(89,7 \%)$ des 204 patients qui sont décédés et 681 (82,7\%) des 823 patients qui étaient vivants au moment du congé.

\section{Utilisation des ressources et soins cliniques}

Comparativement aux patients atteints de l'influenza, ceux atteints de la COVID-19 présentaient un risque plus élevé d'utilisation des ressources des unités de soins intensifs (taux non ajusté $26,4 \%$ C. $18,0 \%, p<0,001$; RR ajusté 1,50 , IC à $95 \%$ $1,25-1,80$ ) et une durée d'hospitalisation accrue (durée médiane non ajustée 8,7 jours c. 4,8 jours, $p<0,001$; taux d'incidence ajusté 1,45 , IC à $95 \% 1,25-1,69$ ) (tableau 3, tableau 4), mais la durée du séjour à l'unité de soins intensifs n'était pas significativement différente après ajustement (tableau 3, tableau 4 et annexe 1, tableau S2).

Les patients atteints de la COVID-19 étaient plus susceptibles d'avoir besoin de ventilation artificielle (18,5\% c. 9,3\%, $p<0,001)$ mais moins susceptibles de subir une bronchoscopie $(2,0 \%$ c. $5,6 \%, p=0,005)$. Les patients atteints de la COVID-19 ont subi au moins une tomodensitométrie thoracique dans $20,4 \%$ des cas et se sont vu prescrire des corticostéroïdes systémiques dans $16,7 \%$ des cas.

\section{Issues stratifiées selon l'âge}

Le taux de mortalité non ajusté chez les patients atteints de la COVID-19 âgés de moins de 50 ans, $50-75$ ans et de plus de 75 ans était de $5,1 \%$, de $13,5 \%$ et de $38,9 \%$, respectivement. Le taux d'utilisation des ressources des unités de soins intensifs pour chaque groupe d'âge était de $29,8 \%$, de $35,2 \%$ et de $11,3 \%$, respectivement, et le taux de réhospitalisation dans les 30 jours était de $9,2 \%$, de $9,9 \%$ et de $7,9 \%$, respectivement (annexe 1, tableau S1).

\section{Scores prédictifs de la mortalité}

La discrimination et le calage des scores pronostiques sont présentés au tableau 5 et à l'annexe 1 , tableau S3, tableau S4 et figure S1. Des données complètes étaient disponibles pour $1 \%$ (ISARIC-4C) à $46 \%$ (Xie) des cas. L'exactitude de la discrimination était maximale pour le score mAPACHE (ASC de 0,86 pour les cas aux données complètes et de 0,81 après répartition), le score CISSS (ASC de 0,83 pour les cas aux données complètes et de 0,80 après répartition) et le score ISARIC-4C (ASC de 0,78 après répartition). Le calage des modèles était peu exact pour les modèles de régression $(\mathrm{Hu}$, Xie et CISSS), tandis que le risque observé augmentait de façon essentiellement linéaire pour les scores à pointage (annexe 1, figure S1).
Les résultats globaux n'ont généralement pas été modifiés par les analyses de sensibilité (voir l'annexe 1 pour de plus amples détails).

\section{Interprétation}

Notre étude contribue à la comparaison entre la COVID-19 et la grippe saisonnière. Il a été avancé que le taux de mortalité de la COVID-19 pourrait être 10 fois supérieur à celui de l'influenza ${ }^{46,47}$, mais ces comparaisons sont indirectes et ont fait l'objet de contestations ${ }^{48}$. Nous avons observé que les patients atteints de la COVID-19 étaient plus susceptibles de décéder (3,5 fois plus à risque), d'être hospitalisés à l'unité de soins intensifs ( 1,5 fois plus à risque) ou de subir une hospitalisation prolongée ( 1,5 fois plus à risque) que les patients atteints de l'influenza, ce qui est comparable aux différences rapportées récemment en France ${ }^{10}$ et aux États-Unis ${ }^{11}$. Par conséquent, les hospitalisations pour cause de COVID-19 sont susceptibles d'avoir des issues significativement plus graves que celles pour cause de grippe saisonnière. Ces différences pourraient avoir été amplifiées par la faible immunité de la population au nouveau coronavirus comparativement au virus de la grippe saisonnière, pour laquelle les patients peuvent avoir une certaine immunité découlant d'infections antérieures et de la vaccination. La gravité relative de la maladie pourrait évoluer à mesure que l'immunité au coronavirus du syndrome respiratoire aigu sévère 2 (SRAS-CoV-2) augmente et que des traitements efficaces sont mis au point.

Au Canada, les hospitalisations pour cause de COVID-19 n'ont pas été bien décrites. Nous avons observé que les deux tiers des patients atteints de la COVID-19 hospitalisés à l'unité de soins intensifs avaient eu besoin d'une ventilation artificielle effractive et que le quart étaient décédés, ce qui est comparable aux résultats de 2 petites études canadiennes sur les unités de soins intensifs ${ }^{6,7}$. Nous ajoutons ici aux données publiées de l'information sur les patients qui n'ont pas été hospitalisés à l'unité de soins intensifs, montrant qu'environ un quart de tous les patients hospitalisés en raison de la COVID-19 et un cinquième de ceux admis au service des urgences ont eu à utiliser les ressources de l'unité des soins intensifs. Par rapport aux données internationales, les patients atteints de la COVID-19 en Ontario (âge médian de 65 ans) étaient légèrement plus âgés que ceux de Chine (âge médian de 51-56 ans) ${ }^{5,46}$ et des États-Unis (âge médian de 62-63 ans) ${ }^{1-3}$, et plus jeunes que ceux du Royaume-Uni (âge médian de 73 ans $)^{4}$, mais les taux de mortalité et d'utilisation des ressources des unités de soins intensifs étaient généralement comparables. Nous avons montré que près de 1 patient atteint de la COVID-19 sur 10 était hospitalisé à nouveau dans les 30 jours suivant son congé, ce qui correspond aux rapports provenant des États-Unis ${ }^{3,50}$.

Comparativement aux patients atteints de l'influenza, ceux atteints de la COVID-19 étaient plus susceptibles d'être des hommes résidant en établissements de soins de longue durée, ce qui correspond aux données probantes qui démontrent que la COVID-19 touche plus gravement les hommes ${ }^{51}$ et a imposé 
Tableau 2 : Signes vitaux, résultats de tests de laboratoire et scores de risque de mortalité chez les patients atteints de la COVID-19 et de l'influenza*

COVID-19

Variable

Température, degrés Celsius

TA systolique, $\mathrm{mm} \mathrm{Hg}$

TA diastolique, $\mathrm{mm} \mathrm{Hg}$

Fréquence cardiaque, battements par minute

Fréquence respiratoire, respirations par minute

Rapport $\mathrm{PaO}_{2} / \mathrm{FiO}_{2}$

Oxygénothérapie d’appoint, $\mathrm{n}^{\text {bre }}(\%) \S$

Hémoglobine, g/L

Hématocrite, L/L

Numération des globules blancs, $\times 10^{9} / \mathrm{L}$

Plaquettes, $\times 10^{9} / \mathrm{L}$

Neutrophiles, $\times 10^{9} / \mathrm{L}$

Lymphocytes, $\times 10^{9} / \mathrm{L}$

Sodium, $\mathrm{mmol} / \mathrm{L}$

Bicarbonate, $\mathrm{mmol} / \mathrm{L}$

Créatinine, $\mu \mathrm{mol} / \mathrm{L}$

Urée, $\mathrm{mmol} / \mathrm{L}$

Albumine, g/L

Bilirubine, $\mu \mathrm{mol} / \mathrm{L}$

Protéine C-réactive, $\mathrm{mg} / \mathrm{L}$

Lactate, $\mathrm{mmol} / \mathrm{L}$

LDH, U/L

D-dimères, $\mu \mathrm{g} / \mathrm{L}$ FEU

Ferritine, $\mu \mathrm{g} / \mathrm{L}$

Glucose, $\mathrm{mmol} / \mathrm{L}$

$\mathrm{PCO}_{2}$ artérielle, $\mathrm{mm} \mathrm{Hg}$

$\mathrm{PO}_{2}$ artérielle, $\mathrm{mm} \mathrm{Hg}$

$\mathrm{PCO}_{2}$ veineuse, $\mathrm{mm} \mathrm{Hg}$

Score de risque de mortalité

$\begin{array}{lc}\text { mAPACHE } & 27(19-34) \\ \text { Score CISSS } & 0,03(0,01-0,06) \\ \text { Score Lu, catégorie 3, } \mathrm{n}^{\text {bre }}(\%) & 581(57) \\ \text { Score Hu } & 0,27(0,08-0,55) \\ \text { Score Xie } & 0,14(0,06-0,26) \\ \text { ISARIC-4C } & 9(7-11) \\ \text { NEWS2 } & 5(2-9)\end{array}$

\section{Influenza}

\begin{tabular}{|c|c|c|c|c|c|}
\hline $\begin{array}{c}\text { Résultat } \\
\text { médian (EI)† }\end{array}$ & $\begin{array}{l}N^{\text {bre }}(\%) \\
\text { effectué } \\
n=1027\end{array}$ & $\begin{array}{c}\text { Résultat } \\
\text { médian (EI)† }\end{array}$ & $\begin{array}{c}N^{\text {bre }}(\%) \\
\text { effectué } \\
n=783\end{array}$ & $\begin{array}{c}\text { DN, } \\
\text { données } \neq\end{array}$ & $\begin{array}{l}\text { DN, N } \text { Nre }^{\text {effectuéł }}\end{array}$ \\
\hline $36,9(36,6-37,5)$ & $1000(97,4)$ & $36,9(36,6-37,4)$ & $578(73,8)$ & 0,07 & 0,71 \\
\hline $126(114-142)$ & $1019(99,2)$ & $129(114-148)$ & $568(72,5)$ & 0,03 & 0,83 \\
\hline $72(64-82)$ & $1019(99,2)$ & $73(65-82)$ & $568(72,5)$ & $<0,01$ & 0,83 \\
\hline $88(76-100)$ & $1019(99,2)$ & 87 (75-99) & $568(72,5)$ & 0,05 & 0,83 \\
\hline $20(18-22)$ & $1017(99,0)$ & $18(18-20)$ & $564(72,0)$ & 0,18 & 0,83 \\
\hline $448(328-462)$ & $1007(98,1)$ & $452(438-467)$ & $551(70,4)$ & 0,40 & 0,82 \\
\hline $458(44,9)$ & $1019(99,2)$ & $212(37,7)$ & $563(71,9)$ & 0,15 & 0,84 \\
\hline $127(111-141)$ & $1006(98,0)$ & $123(107-138)$ & $773(98,7)$ & 0,10 & 0,06 \\
\hline $0,38(0,34-0,42)$ & $1005(98,9)$ & $0,38(0,33-0,42)$ & $773(98,7)$ & 0,06 & 0,07 \\
\hline $7,6(5,5-10,6)$ & $1005(97,9)$ & $7,8(5,5-10,90)$ & $773(98,7)$ & $<0,01$ & 0,07 \\
\hline $214(164-281)$ & $1002(97,6)$ & $186(144-242)$ & $771(98,7)$ & 0,34 & 0,07 \\
\hline $5,6(3,8-8,3)$ & $1002(97,6)$ & $5,7(3,9-8,6)$ & $771(98,7)$ & $<0,01$ & 0,07 \\
\hline $1,0(0,7-1,5)$ & $1000(97,4)$ & $0,9(0,5-1,3)$ & $771(98,7)$ & 0,10 & 0,08 \\
\hline $137(133-140)$ & $1002(97,6)$ & $136(133-139)$ & $775(99,0)$ & 0,18 & 0,11 \\
\hline $25(22-27)$ & $939(91,4)$ & $25(22-27)$ & $763(97,4)$ & 0,01 & 0,27 \\
\hline $89(70-124)$ & $998(97,2)$ & $96(72-135)$ & $770(98,3)$ & 0,07 & 0,08 \\
\hline $6,6(4,4-11,7)$ & $417(40,6)$ & $7,1(4,6-10,6)$ & $343(43,8)$ & 0,08 & 0,07 \\
\hline $32(27-37)$ & $570(55,5)$ & $33(29-37)$ & $448(57,2)$ & 0,15 & 0,04 \\
\hline $9(7-13)$ & $850(82,8)$ & $9(6-14)$ & $599(76,5)$ & 0,01 & 0,16 \\
\hline $77(30-142)$ & $439(42,7)$ & $47(18-100)$ & $59(7,5)$ & 0,31 & 0,89 \\
\hline $1,7(1,3-2,4)$ & $758(73,8)$ & $1,7(1,3-2,5)$ & $605(77,3)$ & 0,05 & 0,09 \\
\hline $315(222-425)$ & $548(53,4)$ & $247(194-364)$ & $152(19,4)$ & 0,03 & 0,75 \\
\hline 1030 (672-1935) & $436(42,5)$ & $911(433-1780)$ & $41(5,2)$ & 0,09 & 0,97 \\
\hline $463(210-1124)$ & $390(38,0)$ & $316(120-675)$ & $84(10,7)$ & 0,12 & 0,67 \\
\hline $6,7(5,8-8,8)$ & $921(89,7)$ & $6,8(5,70-8,7)$ & $727(92,8)$ & 0,01 & 0,11 \\
\hline $43(36-54)$ & $211(20,5)$ & $42(34-49)$ & $116(14,8)$ & 0,21 & 0,15 \\
\hline $73(62-94)$ & $211(20,5)$ & $81(66-126)$ & $116(14,8)$ & 0,36 & 0,15 \\
\hline $43(38-48)$ & $559(54,4)$ & $44(38-50)$ & $449(57,3)$ & 0,13 & 0,06 \\
\hline $27(19-34)$ & S.O. & $28(21-35)$ & S.O. & 0,14 & S.O. \\
\hline $0,03(0,01-0,06)$ & S.O. & $0,03(0,02-0,06)$ & S.O. & 0,05 & S.O. \\
\hline $581(57)$ & S.O. & $515(66)$ & S.O. & 0,19 & S.O. \\
\hline $0,27(0,08-0,55)$ & S.O. & $0,27(0,10-0,51)$ & S.O. & 0,05 & S.O. \\
\hline $0,14(0,06-0,26)$ & S.O. & $0,16(0,07-0,27)$ & S.O. & 0,04 & S.O. \\
\hline $9(7-11)$ & S.O. & $9(8-11)$ & S.O. & 0,08 & S.O. \\
\hline $5(2-9)$ & S.O. & $4(1-6)$ & S.O. & 0,52 & S.O. \\
\hline
\end{tabular}

Remarque : CISSS = système de classification des maladies graves (critical illness severity scoring system), COVID-19= maladie à coronavirus 2019, DN = différence normalisée, $\mathrm{EI}=$ écart interquartile, FEU = équivalent fibrinogène (valeurs uniformisées à cette unité entre les sites si les unités de mesure sont différentes), ISARIC-4C = ISARIC Coronavirus Clinical Characterisation Consortium $4 \mathrm{C}, \mathrm{LDH}=$ lactate déshydrogénase, $\mathrm{mAPACHE}=$ score Acute Physiology and Chronic Health Evaluation modifié, NEWS2 = échelle nationale d'alerte précoce, Rapport $\mathrm{SO}_{2} / \mathrm{FiO}_{2}=$ rapport de la saturation en oxygène et de la fraction inspirée en oxygène, $\mathrm{S} . \mathrm{O} .=$ sans objet, $\mathrm{TA}=$ tension artérielle.

*Nous présentons le premier résultat de tests de laboratoire et la première mesure de signes vitaux valides recueillis entre le triage au service des urgences et $72 \mathrm{~h}$ après

l'hospitalisation. Les signes vitaux ne sont pas enregistrés sous forme numérique de façon systématique dans tous les centres hospitaliers lorsque les patients sont au service des urgences ou dans une unité de soins intensifs. Pour les patients atteints de la COVID-19, mais pas pour ceux atteints de l'influenza, les signes vitaux ont été extraits de façon manuelle des dossiers médicaux pour la période entre le triage du service des urgences et $24 \mathrm{~h}$ après l'hospitalisation (afin de calculer les scores du risque de mortalité). Les scores du risque de mortalité ${ }^{31-36,39}$ ont été calculés sur la base de la première mesure valide entre le triage du service des urgences et 24 heures après l'hospitalisation; les valeurs moyennes imputées étaient manquantes (annexe 1). L'intervalle de points possible pour les scores basés sur des points était les suivants : mAPACHE (0-159), Lu (1-3), ISARIC-4C (0-21), NEWS2 (0-20). Les autres scores sont basés sur les probabilités (échelle de scores comprise entre 0 et 1 ). On calcule les scores de risque présentés dans le présent tableau selon l'imputation moyenne des données de laboratoire manquantes. On calcule les scores ISARIC-4C et NEWS2 uniquement pour les cas où les données sur l'état mental sont disponibles ( $n=242$ hospitalisations en raison de la COVID-19 et $n=46$ en raison de l'influenza).

†Sauf indication contraire.

łUne DN supérieure à 0,1 indique un déséquilibre entre les groupes.

§L’oxygénothérapie d'appoint correspond au nombre de patients qui nécessitent un supplément d'oxygène ou une ventilation artificielle. 


\section{$\mathbf{N}^{\text {bre }}(\%)$ d'hospitalisationt}

\begin{tabular}{|c|c|c|c|}
\hline Variable & $\begin{array}{l}\text { COVID-19 } \\
n=1027\end{array}$ & $\begin{array}{c}\text { Influenza } \\
n=783\end{array}$ & valeur $p$ \\
\hline Décès & $204(19,9)$ & $48(6,1)$ & $<0,001$ \\
\hline Réadmission dans les 7 joursł & $32(4,3)$ & $22(3,1)$ & 1,0 ฯ \\
\hline Réadmission dans les 30 jours§ & $58(9,3)$ & $69(9,6)$ & 0,9 \\
\hline Utilisation des ressources des unités de soins intensifs & $271(26,4)$ & $141(18,0)$ & $<0,001$ \\
\hline Durée médiane du séjour hospitalier, jours (EI) & $8,7(3,6-18,9)$ & $4,8(2,3-10,4)$ & $<0,001$ \\
\hline Durée médiane du séjour à l'unité de soins intensifs, jours (EI) & $10,9(4,0-17,8)$ & $6,0(2,3-13,0)$ & $<0,001$ \\
\hline Durée médiane du séjour au service d’urgence, heures (EI) & $8,7(6,1-13,2)$ & $21,1(12,0-32,2)$ & $<0,0019$ \\
\hline Ventilation mécanique effractive & $190(18,5)$ & $73(9,3)$ & $<0,0019$ \\
\hline Endoscopie gastro-intestinale & $21(2,0)$ & $27(3,4)$ & 1,0 ฯ \\
\hline Bronchoscopie & $21(2,0)$ & $44(5,6)$ & 0,0059 \\
\hline Dialyse $e^{\star \star}$ & $79(7,7)$ & $43(5,5)$ & 1,0 ฯ \\
\hline TDM thoracique & $210(20,4)$ & $168(21,5)$ & 1,0 ฯ \\
\hline Antibiotiques respiratoires†† & $730(71,6)$ & $599(77,1)$ & 0,69 \\
\hline Corticostéroïdes & $170(16,7)$ & $284(36,6)$ & $<0,0019$ \\
\hline Warfarine ou ACOD & $157(15,4)$ & $156(20,1)$ & 0,69 \\
\hline
\end{tabular}

Remarque : $\mathrm{ACOD}$ = anticoagulant oral direct, COVID-19 = maladie à coronavirus 2019, IE = écart interquartile, TDM = tomodensitométrie.

*On déclare la réadmission dans une unité médicale ou une unité de soins intensifs médicaux ou chirurgicaux de l'un des centres hospitaliers participants chez les patients qui ont obtenu leur congé de leur vivant et pour les consultations qui pourraient être associées entre elles par un numéro d'assurance-santé valide. Pour les ressources hospitalières et les soins cliniques, nous indiquons le nombre de patients recevant au moins l'un des soins décrits.

†Sauf indication contraire.

‡Après avoir exclu les patients décédés et ceux qui ont obtenu leur congé au cours des 7 derniers jours de l'étude, le dénominateur était de 745 hospitalisations pour la CoVID-19 et de 720 pour l'influenza.

$\S$ Après avoir exclu les patients décédés et ceux qui ont obtenu leur congé au cours des 30 derniers jours de l'étude, le dénominateur était de 625 hospitalisations pour la COVID-19 et de 718 pour l'influenza.

ILes valeurs $p$ ont été ajustées à l'aide de la correction de Bonferroni pour les 10 issues secondaires.

${ }^{* \star}$ La dialyse comprend l'hémodialyse et la dialyse péritonéale, et comprend à la fois l'emploi chronique et les nouvelles initiatives thérapeutiques.

††Les antibiotiques respiratoires comprennent tous ceux énumérés à l’annexe 1.

Tableau 4 : Issues cliniques des patients atteints de la COVID-19 comparativement à celles des patients atteints de l'influenza, avant et après un ajustement à variables multiples*

\begin{tabular}{|lcc|}
\hline Issue & $\begin{array}{c}\text { Effet non ajusté } \\
\text { (IC à 95\%) }\end{array}$ & $\begin{array}{c}\text { Effet ajusté } \dagger \\
\text { (IC à 95\%) }\end{array}$ \\
\hline Décès & $3,24(2,40-4,38)$ & $3,46(2,56-4,68)$ \\
\hline $\begin{array}{l}\text { Utilisation des ressources des unités de soins } \\
\text { intensifs }\end{array}$ & $1,47(1,22-1,76)$ & $1,50(1,25-1,80)$ \\
\hline Réadmission dans les 30 jours & $0,97(0,69-1,35)$ & $0,98(0,70-1,39)$ \\
\hline Durée du séjour hospitalier & $1,31(1,09-1,58)$ & $1,45(1,25-1,69)$ \\
\hline Durée du séjour à l'USI & $0,93(0,57-1,52)$ & $1,25(0,92-1,70)$ \\
\hline
\end{tabular}

Remarque : COVID-19 = maladie à coronavirus 2019, IC = intervalle de confiance, USI = unité de soins intensifs.

*Les modèles ont été ajustés selon l'âge du patient, le sexe, l'établissement de soins de longue durée, le score de comorbidité de Charlson, le centre hospitalier où le patient est admis, ainsi que le quintile de revenu et le quintile de la proportion de la population qui s'identifie comme appartenant à une minorité visible du quartier du patient. Les issues cliniques à l'étude sont : la mortalité perhospitalière, l'hospitalisation à l'unité de soins intensifs à tout moment au cours du séjour hospitalier, la réadmission au sein d'une unité médicale ou d'une unité de soins intensifs médicaux ou chirurgicaux de l'un des centres hospitaliers participants dans les 30 jours suivant le congé, la durée du séjour hospitalier et la durée du séjour à l'unité de soins intensifs.

tLes modèles de régression de Poisson ont été ajustés selon les décès, l'hospitalisation à l'unité de soins intensifs et les réadmissions (effet = risque relatif) et les modèles de régression binomiale négative ont été ajustés pour la durée de séjour hospitalier et la durée de séjour à l'unité de soins intensifs (effet = taux d'incidence). un lourd fardeau aux établissements de soins de longue durée ontariens ${ }^{43,52}$. Les patients issus de quartiers à faible revenu étaient surreprésentés dans les groupes de COVID-19 et de l'influenza, nous rappelant que les inégalités socioéconomiques observées dans le contexte de la COVID-1953 correspondent à celles qu'on observe pour d'autres maladies, dont l'influenza $a^{54}$. Notons que la majorité des patients atteints de la COVID-19 qui ont été hospitalisés avaient peu de comorbidités (score de Charlson de 0 ), et un cinquième d'entre eux avaient moins de 50 ans. L'utilisation des ressources des unités de soins intensifs était fréquente dans le groupe 
le plus jeune, ce qui vient confirmer que la COVID-19 peut gravement toucher les personnes plus jeunes et celles qui présentent relativement peu de comorbidités.

Les prédictions de la mortalité dans le contexte de la COVID-19 peuvent éclairer le processus de décision clinique et l'affectation des ressources. De nombreux scores prédictifs ont été signalés ${ }^{38}$, mais plusieurs ont été élaborés à partir de petites cohortes et nécessitent une validation externe. Nous avons déterminé que le modèle mAPACHE présente la meilleure exactitude discriminative et un bon calage. Il offre aussi l'avantage d'un calcul automatique dans les dossiers médicaux électroniques. Les cliniciens au chevet des patients peuvent facilement calculer les scores ISARIC-4C et NEWS2, qui offrent une discrimination relativement exacte et un calage adéquat. La performance des modèles NEWS2, Hu, Lu et Xie au sein de notre cohorte était comparable à celle observée dans une étude de validation comprenant 411 patients menée dans un seul centre au Royaume-Uni ${ }^{37}$, et la performance du modèle ISARIC-4C était comparable à celle mentionnée dans sa description originale ${ }^{39}$, renforçant la confiance dans nos estimations de performance des modèles. Cependant, peu de dossiers d'hospitalisation comprenaient des données complètes, principalement en raison de l'absence de tests de laboratoire. Par exemple, le score ISARIC-4C tient compte des résultats d'analyse de l'urée, laquelle a été demandée dans moins de $11 \%$ des cas de COVID-19 dans 4 centres hospitaliers en raison de son utilisation restreinte à la suite d'initiatives de gestion des ressources. Ainsi, le calcul de certains scores pourrait exiger des changements à la pratique clinique courante, bien que la performance des modèles demeure raisonnable avec une simple imputation moyenne des valeurs manquantes. La saisie non uniforme de l'état de santé mentale (manquante dans $70 \%$ des hospitalisations) a limité le nombre d'hospitalisations pour lesquelles nous avons pu valider les modèles NEWS2 et ISARIC-4C, mais cela ne devrait pas entraver l'utilisation de ces outils dans la pratique clinique. Notre étude offre une solide validation externe que le risque de décès des suites de la COVID-19 peut être prédit de façon raisonnable avec de simples scores. L'élaboration de modèles prédictifs pour le contexte canadien, par exemple en adaptant des scores conçus à l'étranger, sera un sujet de recherche important pour des travaux futurs.

\section{Limites de l'étude}

Notre étude présente toutefois plusieurs limites. Premièrement, nous avons inclus 7 centres hospitaliers universitaires d'envergure qui acceptaient les transferts de patients atteints de la COVID-19 à l'unité des soins intensifs. Pour éviter de surestimer la gravité de la COVID-19, nous avons reproduit nos analyses avec des patients hospitalisés depuis le service des urgences afin d'exclure les transferts entre établissements, et nos résultats sont demeurés cohérents. Nous croyons que nos conclusions sont généralisables, puisque l'incidence de la mortalité observée dans notre cohorte était comparable à celle signalée dans de grandes études réalisées aux États-Unis ${ }^{1}$ et au Royaume-Uni ${ }^{4}$, et que notre étude comprenait environ $25 \%$ des patients hospitalisés pour la COVID-19 en Ontario. Deuxièmement, nous avons recueilli uniquement les données saisies de façon systématique dans les sources administratives ou le dossier médical électronique; il n'a donc pas été possible de tenir compte des préférences des patients ou d'ajuster les données en fonction de celles-ci en ce qui a trait aux soins intensifs, aux symptômes présentés ou à certains facteurs de risques importants comme l'obésité ou le tabagisme. Nos analyses du revenu et du statut de minorité visible n'ont pu être réalisées qu'à l'échelle du quartier; elles n'offrent donc pas une granularité suffisante pour tirer des conclusions solides. Troisièmement, nous rapportons la réhospitalisation dans les 30 jours pour tous les centres hospitaliers participants. Bien que les réhospitalisations soient possiblement sousestimées, $82 \%$ de celles survenues dans notre région se sont produites au centre hospitalier d'origine ${ }^{55}$, et nous avons été en mesure de capturer aussi celles survenues dans d'autres centres hospitaliers participants. Quatrièmement, la gravité de la grippe saisonnière varie chaque année, et nous avons seulement intégré les données de l'année 2019-2020. Cependant, dans notre étude, le taux de mortalité associé aux hospitalisations dues à l'influenza $(6,1 \%)$ correspond aux taux de mortalité d'environ $3 \%$ à $6 \%$ relevé dans une revue systématique examinant plus de 120000 hospitalisations liées à l'influenza ${ }^{56}$, et nos résultats sont comparables à ceux publiés dans des études récentes réalisées en France $^{10}$ et aux États-Unis ${ }^{11}$. Cinquièmement, l'utilisation accrue de la dexaméthasone et d'autres traitements contre la COVID-19 depuis la fin de la période couverte par notre étude pourrait modifier les estimations des taux de mortalité et l'exactitude des scores prédictifs. Une validation tenant compte des traitements les plus récents serait utile. Enfin, nous avons été incapables d'obtenir des données sur la cause du décès, lesquelles peuvent être peu fiables lorsqu'obtenues de sources administratives ${ }^{57}$; de ce fait, nous ne pouvons pas rapporter le nombre de patients dont le décès a été causé directement par la COVID-19. Les diagnostics principaux au moment du congé étaient la COVID-19, une pneumonie virale, le sepsis ou des soins palliatifs chez $89,7 \%$ des patients décédés qui étaient atteints de la COVID-19, laissant sous-entendre que la plupart de ces décès sont probablement attribuables à la COVID-19.

\section{Conclusion}

Les adultes atteints de la COVID-19 hospitalisés dans 7 centres hospitaliers ontariens au cours de la première vague de la pandémie ont eu besoin de ressources hospitalières substantielles et ont connu un taux de mortalité élevé. Ces patients présentaient des taux accrus de mortalité, d'utilisation des ressources des unités de soins intensifs et de ventilation artificielle effractive, et nécessitaient un séjour à l'hôpital plus long que les patients hospitalisés en raison de l'influenza. On peut prédire avec une exactitude raisonnable le risque de mortalité chez les patients hospitalisés atteints de COVID-19 à l'aide de simples scores. 


\begin{tabular}{|c|c|c|c|c|c|c|}
\hline Score & $\begin{array}{l}\text { Méthode de } \\
\text { calcul }\end{array}$ & Prédicteurs & $\begin{array}{c}\mathrm{N}^{\text {bre }}(\%) \\
\text { d'hospitalisations }^{\text {avec données }} \\
\text { complètes } \\
n=1027\end{array}$ & $\begin{array}{c}\text { ASC, } \\
\text { données } \\
\text { complètes } \\
\text { (IC à } 95 \% \text { ) }\end{array}$ & $\begin{array}{c}\mathrm{N}^{\text {bre }}(\%) \\
\text { d'hospitalisations } \\
\text { après imputation } \\
n=1027\end{array}$ & $\begin{array}{l}\text { ASC après } \\
\text { imputation } \\
\text { (IC à } 95 \% \text { ) }\end{array}$ \\
\hline Lu & $\begin{array}{l}\text { Système de } \\
\text { points }\end{array}$ & Âge, protéine C-réactive & $390(37,9)$ & $\begin{array}{c}0,71 \\
(0,66-0,76)\end{array}$ & $1027(100)$ & $\begin{array}{c}0,68 \\
(0,65-0,71)\end{array}$ \\
\hline Score Hu & $\begin{array}{l}\text { Système de } \\
\text { régression }\end{array}$ & $\begin{array}{l}\text { Âge, protéine C-réactive, } \\
\text { D-dimères, lymphocytes }\end{array}$ & $230(22,4)$ & $\begin{array}{c}0,78 \\
(0,70-0,86)\end{array}$ & $1027(100)$ & $\begin{array}{c}0,72 \\
(0,68-0,76)\end{array}$ \\
\hline Score Xie & $\begin{array}{l}\text { Système de } \\
\text { régression }\end{array}$ & $\begin{array}{l}\text { Âge, LDH, lymphocyte, saturation } \\
\text { en oxygène }\end{array}$ & $469(45,6)$ & $\begin{array}{c}0,80 \\
(0,75-0,85)\end{array}$ & $972(94,6)$ & $\begin{array}{c}0,75 \\
(0,71-0,79)\end{array}$ \\
\hline ISARIC-4C & $\begin{array}{l}\text { Système de } \\
\text { points }\end{array}$ & $\begin{array}{l}\text { Âge, sexe, score de comorbidités } \\
\text { de Charlson, fréquence } \\
\text { respiratoire, saturation en } \\
\text { oxygène, score sur l'échelle de } \\
\text { Glasgow, urée, protéine C-réactive }\end{array}$ & $12(1,2)$ & S.O. ${ }^{\dagger}$ & $242(23,6)$ & $\begin{array}{c}0,78 \\
(0,70-0,85)\end{array}$ \\
\hline mAPACHE & $\begin{array}{l}\text { Système de } \\
\text { points }\end{array}$ & $\begin{array}{l}\text { Âge, ventilation artificielle, } \\
\text { hématocrite, leucocytes, sodium, } \\
\text { glucose, bilirubine, urée, créatine, } \\
\text { température, fréquence } \\
\text { cardiaque, fréquence respiratoire, } \\
\text { pression artérielle moyenne, } \\
\text { cancer métastatique, sida, } \\
\text { insuffisance hépatique, cirrhose, } \\
\text { leucémie, lymphome, } \\
\text { immunosuppression, myélome } \\
\text { multiple }\end{array}$ & $348(33,9)$ & $\begin{array}{c}0,86 \\
(0,83-0,90)\end{array}$ & $976(95,0)$ & $\begin{array}{c}0,81 \\
(0,78-0,85)\end{array}$ \\
\hline $\begin{array}{l}\text { Score } \\
\text { CISSS }\end{array}$ & $\begin{array}{l}\text { Système de } \\
\text { régression }\end{array}$ & $\begin{array}{l}\text { Âge, ventilation artificielle, } \\
\text { intervention chirurgicale, } \\
\text { hématocrite, leucocytes, sodium, } \\
\text { glucose, bilirubine, créatine, } \\
\text { bicarbonate, albumine, } \\
\text { température, fréquence } \\
\text { cardiaque, fréquence respiratoire, } \\
\text { pression artérielle moyenne, } \\
\text { cancer métastatique, sida, } \\
\text { insuffisance hépatique, cirrhose, } \\
\text { leucémie, lymphome, } \\
\text { immunosuppression, myélome } \\
\text { multiple }\end{array}$ & $357(34,8)$ & $\begin{array}{c}0,83 \\
(0,78-0,88)\end{array}$ & $976(95,0)$ & $\begin{array}{c}0,80 \\
(0,77-0,84)\end{array}$ \\
\hline NEWS2 & $\begin{array}{l}\text { Système de } \\
\text { points }\end{array}$ & $\begin{array}{l}\text { Fréquence respiratoire, saturation } \\
\text { en oxygène, pression artérielle } \\
\text { systolique, fréquence cardiaque, } \\
\text { état de conscience/nouvel état de } \\
\text { confusion, température }\end{array}$ & $242(23,6)$ & $\begin{array}{c}0,72 \\
(0,63-0,80)\end{array}$ & $242(23,6)$ & $\begin{array}{c}0,72 \\
(0,63-0,80)\end{array}$ \\
\hline
\end{tabular}

Remarque : $\mathrm{ASC}=$ aire sous la courbe de la fonction d'efficacité du récepteur, CISSS = système de classification des maladies graves (critical illness severity scoring system), COVID-19 = maladie à coronavirus 2019, GCS = score de Glasgow, IC = intervalle de confiance, ISARIC-4C = ISARIC Coronavirus Clinical Characterisation Consortium 4C, mAPACHE = score Acute Physiology and Chronic Health Evaluation modifié, NEWS2 = échelle nationale d'alerte précoce (National Early Warning Score), S.O. = sans objet. *Les scores du risque de mortalité ${ }^{31-36,39}$ ont été calculés sur la base de la première mesure valide entre le triage au service des urgences et 24 heures après l'hospitalisation. Nous rapportons la performance du modèle selon les hospitalisations comprenant des données complètes pour chacune des entrées et après une supposition moyenne pour les données de laboratoire manquantes (voir l'annexe 1 pour de plus amples détails). Nous n'avons pas estimé les données manquantes pour les signes vitaux ou l'état mental ou neurologique, car nous ne pensions pas qu'il était raisonnable de supposer que ces valeurs soient normales. Le taux de mortalité parmi les 242 hospitalisations utilisées pour la validation des scores ISARIC-4C et NEWS2 était de $17,4 \%$ (42 décès).

${ }^{\dagger}$ Nombre d'échantillons insuffisant pour procéder au calcul.

\section{Références}

1. Richardson S, Hirsch JS, Narasimhan M, et al. Presenting characteristics, comorbidities, and outcomes among 5700 patients hospitalized with COVID-19 in the New York City area [erratum publié dans JAMA 2020;323:2098]. JAMA 2020;323:2052-9.

2. Goyal P, Choi JJ, Pinheiro LC, et al. Clinical characteristics of COVID-19 in New York City. N Engl J Med 2020;382:2372-4.
3. Chopra V, Flanders SA, O'Malley M, et al. Sixty-day outcomes among patients hospitalized with COVID-19. Ann Intern Med 2020 Nov. 11 [cyberpublication avant impression]. doi: 10.7326/M20-5661.

4. Docherty AB, Harrison EM, Green CA, et al.; ISARIC4C investigators. Features of 20133 UK patients in hospital with COVID-19 using the ISARIC WHO Clinical Characterisation Protocol: prospective observational cohort study. BMJ 2020;369:m1985.

5. Zhou F, Yu T, Du R, et al. Clinical course and risk factors for mortality of adult 
inpatients with COVID-19 in Wuhan, China: a retrospective cohort study [erratum publié dans Lancet 2020;395:1038]. Lancet 2020;395:1054-62.

6. Mitra AR, Fergusson NA, Lloyd-Smith E, et al. Baseline characteristics and outcomes of patients with COVID-19 admitted to intensive care units in Vancouver, Canada: a case series. CMAJ 2020;192:E694-701.

7. Cavayas YA, Noël A, Brunette V, et al. Early experience with critically ill patients with COVID-19 in Montreal. Can J Anaesth 2021;68:204-13.

8. Mortality analyses. Baltimore: Johns Hopkins University \& Medicine, Coronavirus Resource Centre. Accessible ici : https://coronavirus.jhu.edu/data/mortality (consulté le 5 déc. 2020).

9. Tolksdorf K, Buda S, Schuler E, et al. Influenza-associated pneumonia as reference to assess seriousness of coronavirus disease (COVID-19). Euro Surveill 2020;25:2000258.

10. Piroth L, Cottenet J, Mariet A-S, et al. Comparison of the characteristics, morbidity, and mortality of COVID-19 and seasonal influenza: a nationwide, population-based retrospective cohort study. Lancet Respir Med 2020 Dec. 17 [cyberpublication avant impression]. doi: 10.1016/S2213-2600(20)30527-0.

11. Xie Y, Bowe B, Maddukuri G, et al. Comparative evaluation of clinical manifestations and risk of death in patients admitted to hospital with COVID-19 and seasonal influenza: cohort study. BMJ 2020;371:m4677.

12. Verma AA, Guo Y, Kwan JL, et al. Patient characteristics, resource use and outcomes associated with general internal medicine hospital care: the General Medicine Inpatient Initiative (GEMINI) retrospective cohort study. CMAJ Open 2017;5:E842-9.

13. Verma AA, Pasricha SV, Jung HY, et al. Assessing the quality of clinical and administrative data extracted from hospitals: the General Medicine Inpatient Initiative (GEMINI) experience. J Am Med Inform Assoc 2020 Nov. 4 [cyberpublication avant impression]. doi: 10.1093/jamia/ocaa225.

14. ICD-10-CA coding direction for suspected COVID-19 cases. Ottawa: Canadian Institute for Health Information; 2020. Accessible ici : www.cihi.ca/en/bulletin/ icd-10-ca-coding-direction-for-suspected-covid-19-cases (consulté le 19 nov. 2020).

15. Kadri SS, Gundrum J, Warner S, et al. Uptake and accuracy of the diagnosis code for COVID-19 among US hospitalizations. JAMA 2020;324:2553-4.

16. Hamilton MA, Calzavara A, Emerson SD, et al. Validating International Classification of Disease 10th Revision algorithms for identifying influenza and respiratory syncytial virus hospitalizations. PLoS One 2021;16:e0244746.

17. Hossein $\mathrm{H}$, Ali KM, Hosseini M, et al. Value of chest computed tomography scan in diagnosis of COVID-19; a systematic review and meta-analysis. Clin Trans/ Imaging 2020 Oct. 12 [cyberpublication avant impression]. doi: 10.1007/ s40336-020-00387-9.

18. Mandell LA, Wunderink RG, Anzueto A, et al.; Infectious Diseases Society of America. American Thoracic Society. Infectious Diseases Society of America/ American Thoracic Society consensus guidelines on the management of community-acquired pneumonia in adults. Clin Infect Dis 2007;44:S27-72.

19. Metlay JP, Waterer GW, Long AC, et al. Diagnosis and treatment of adults with community-acquired pneumonia. An official clinical practice guideline of the American Thoracic Society and Infectious Diseases Society of America. Am J Respir Crit Care Med 2019;200:e45-67.

20. Dragan $\mathrm{V}$, Wei $\mathrm{Y}$, Elligsen $\mathrm{M}$, et al. Prophylactic antimicrobial therapy for acute aspiration pneumonitis. Clin Infect Dis 2018;67:513-8.

21. RECOVERY Collaborative Group; Horby P, Lim WS, Emberson JR, et al. Dexamethasone in hospitalized patients with COVID-19: preliminary report. $N$ Engl J Med 2020 July 17 [cyberpublication avant impression]. doi: 10.1056/ NEJMoa2021436.

22. Godoy LC, Goligher EC, Lawler PR, et al. Anticipating and managing coagulopathy and thrombotic manifestations of severe COVID-19. CMAJ 2020; 192:E1156-61.

23. Blum D, Meraz-Munoz A, Harel Z. Kidney injury associated with COVID-19. CMAJ 2020;192:E1065.

24. Tse F, Borgaonkar M, Leontiadis GI. COVID-19: advice from the Canadian Association of Gastroenterology for endoscopy facilities, as of March 16, 2020. J Can Assoc Gastroenterol 2020;3:147-9.

25. Wahidi MM, Shojaee S, Lamb CR, et al. The use of bronchoscopy during the coronavirus disease 2019 pandemic. Chest 2020;158:1268-81.

26. Healthcare Cost and Utilization Project (HCUP). Clinical Classifications Software Refined (CCSR). Rockville (MD): Agency for Healthcare Research and Quality; 2020. Accessible ici : www.hcup-us.ahrq.gov/toolssoftware/ccsr/ccs_ refined.jsp (consulté le 10 déc. 2020).

27. Verma AA, Guo Y, Kwan JL, et al. Prevalence and costs of discharge diagnoses in inpatient general internal medicine: a multi-center cross-sectional study. J Gen Intern Med 2018;33:1899-904.

28. Quan H, Li B, Couris CM, et al. Updating and validating the charlson comorbidity index and score for risk adjustment in hospital discharge abstracts using data from 6 countries. Am J Epidemiol 2011;173:676-82.
29. Postal Code Conversion File Plus (PCCF+). Ottawa: Statistics Canada. Accessible ici : www150.statcan.gc.ca/n1/en/catalogue/82F0086X (consulté le 20 janv. 2021).

30. Measuring health inequalities: a toolkit - area-level equity stratifiers using PCCF and PCCF+. Ottawa: Canadian Institute for Health Information; 2018. Accessible ici : www.cihi.ca/sites/default/files/document/cphi-toolkit-area-level -measurement-pccf-2018-en-web.pdf (consulté le 20 janv. 2021).

31. Fortis S, O'Shea AMJ, Beck BF, et al. An automated computerized critical illness severity scoring system derived from APACHE III: modified APACHE. J Crit Care 2018;48:237-42.

32. Fortis S, O'Shea AMJ, Beck MAEBF, et al. A simplified critical illness severity scoring system (CISSS): development and internal validation. J Crit Care 2021;61:21-8.

33. Lu J, Hu S, Fan R, et al. ACP risk grade: a simple mortality index for patients with confirmed or suspected severe acute respiratory syndrome coronavirus 2 disease (COVID-19) during the early stage of outbreak in Wuhan, China. medRxiv 2020 Feb. 23. doi: 10.1101/2020.02.20.20025510.

34. Hu C, Liu Z, Jiang Y, et al. Early prediction of mortality risk among patients with severe COVID-19, using machine learning. Int J Epidemiol 2021;49:1918-29.

35. Xie J, Hungerford D, Chen $\mathrm{H}$, et al. Development and external validation of a prognostic multivariable model on admission for hospitalized patients with COVID-19. medRxiv 2020 Apr. 7. doi: 10.1101/2020.03.28.20045997.

36. National Early Warning Score (NEWS) 2. London (UK): Royal College of Physicians. Accessible ici : www.rcplondon.ac.uk/projects/outputs/national-early -warning-score-news-2 (consulté le 20 janv. 2021).

37. Gupta RK, Marks M, Samuels THA, et al.; UCLH COVID-19 Reporting Group. Systematic evaluation and external validation of 22 prognostic models among hospitalised adults with COVID-19: an observational cohort study. Eur Respir J 2020;56:2003498.

38. Wynants L, Van Calster B, Collins GS, et al. Prediction models for diagnosis and prognosis of COVID-19 infection: systematic review and critical appraisal [erratum publié dans BMJ 2020;369:m2204]. BMJ 2020;369:m1328.

39. Knight SR, Ho A, Pius R, et al.; ISARIC4C investigators. Risk stratification of patients admitted to hospital with covid-19 using the ISARIC WHO Clinical Characterisation Protocol: development and validation of the 4C Mortality Score [erratum publié dans BMJ 2020;371:m4334]. BMJ 2020;370:m3339.

40. Austin PC. Using the standardized difference to compare the prevalence of a binary variable between two groups in observational research. Commun Stat Simul Comput 2009;38:1228-34.

41. Clift AK, Coupland CAC, Keogh $\mathrm{RH}$, et al. Living risk prediction algorithm (QCOVID) for risk of hospital admission and mortality from coronavirus 19 in adults: national derivation and validation cohort study. BMJ 2020;371:m3731.

42. Tai DBG, Shah A, Doubeni CA, et al. The disproportionate impact of COVID-19 on racial and ethnic minorities in the United States. Clin Infect Dis 2020 June 20 [cyberpublication avant impression]. doi: 10.1093/cid/ciaa815.

43. Liu M, Maxwell CJ, Armstrong P, et al. COVID-19 in long-term care homes in Ontario and British Columbia. CMAJ 2020;192:E1540-6.

44. Zou G. A modified poisson regression approach to prospective studies with binary data. Am J Epidemiol 2004;159:702-6.

45. Ontario Agency for Health Protection and Promotion (Public Health Ontario). Daily epidemiologic summary: COVID-19 in Ontario - January 15, 2020 to June 30, 2020. Toronto: Queen's Printer for Ontario; 2020. Accessible ici : https://files. ontario.ca/moh-covid-19-report-en-2020-07-01.pdf (consulté le 20 janv. 2021).

46. Brazeau NF, Verity R, Jenks S, et al. Report 34: COVID-19 infection fatality ratio estimates from seroprevalence. London (UK): MRC Centre for Global Infectious Disease Analysis, School of Public Health, Imperial College London; 2020:1-18. Accessible ici : www.imperial.ac.uk/mrc-global-infectious-disease-analysis/ covid-19/report-34-ifr/ (consulté le 28 janv. 2021).

47. Pastor-Barriuso R, Pérez-Gómez B, Hernán MA, et al. Infection fatality risk for SARS-CoV-2 in community dwelling population of Spain: nationwide seroepidemiological study. BMJ 2020;371:m4509.

48. Ioannidis JPA, Axfors C, Contopoulos-loannidis DG. Population-level COVID-19 mortality risk for non-elderly individuals overall and for non-elderly individuals without underlying diseases in pandemic epicenters. Environ Res 2020;188:109890.

49. Wu C, Chen X, Cai Y, et al. Risk factors associated with acute respiratory distress syndrome and death in patients with coronavirus disease 2019 pneumonia in Wuhan, China. JAMA Intern Med 2020;180:934-43.

50. Lavery AM, Preston LE, Ko JY, et al. Characteristics of hospitalized COVID-19 patients discharged and experiencing same-hospital readmission: United States, March-August 2020. MMWR Morb Mortal Wkly Rep 2020;69:1695-9.

51. Takahashi T, Ellingson MK, Wong $P$, et al. Sex differences in immune responses that underlie COVID-19 disease outcomes. Nature 2020;588:315-20.

52. Fisman DN, Bogoch I, Lapointe-Shaw L, et al. Risk factors associated with mortality among residents with coronavirus disease 2019 (COVID-19) in long-term care facilities in Ontario, Canada. JAMA Netw Open 2020;3:e2015957. 
53. Niedzwiedz CL, O’Donnell CA, Jani BD, et al. Ethnic and socioeconomic differences in SARS-CoV-2 infection: prospective cohort study using UK Biobank. BMC Med 2020;18:160.

54. Sloan C, Chandrasekhar R, Mitchel E, et al. Socioeconomic disparities and influenza hospitalizations, Tennessee, USA. Emerg Infect Dis 2015;21:1602-10.

55. Staples JA, Thiruchelvam D, Redelmeier DA. Site of hospital readmission and mortality: a population-based retrospective cohort study. CMAJ Open 2014;2:E77-85.
56. Pormohammad A, Ghorbani S, Khatami A, et al. Comparison of influenza type A and B with COVID-19: a global systematic review and meta-analysis on clinical, laboratory and radiographic findings. Rev Med Virol 2020 Oct. 9 [cyberpublication avant impression]. doi: 10.1002/rmv.2179.

57. Mikkelsen L, Iburg KM, Adair T, et al. Assessing the quality of cause of death data in six high-income countries: Australia, Canada, Denmark, Germany, Japan and Switzerland. Int J Public Health 2020;65:17-28.
Intérêts concurrents : Amol Verma et Fahad Razak déclarent des honoraires personnels de Santé Ontario. Michael Fralick déclare des honoraires personnels de Pine Trees, en dehors du travail soumis. Aucun autre intérêt concurrent n'a été déclaré.

Cet article a été révisé par des pairs.

Affiliations : Institut du savoir Li Ka Shing (Verma, Hora, Jung, Chan, Mamdani, Razak), Hôpital St. Michael, Unity Health Toronto; Département de médecine (Verma, Fralick, Malecki, Lapointe-Shaw, Weinerman, Tang, Kwan, Liu, Rawal, Cheung, Herridge, Mamdani, Razak) et Institut des politiques, de la gestion et de l'évaluation de la santé (Verma, Cheung, Mamdani, Razak), Université de Toronto, Toronto, Ont.; Département de géographie et de gestion environnementale (Hora), Université de Waterloo, Waterloo, Ont.; Département de médecine (Fralick, Kwan), Système de santé Sinai; Département de médecine (Lapointe-Shaw, Liu, Rawal, Cheung, Herridge) et Institut de recherche de l'Hôpital général de Toronto (Lapointe-Shaw), Réseau universitaire de santé; Women's Institute for Health System Solutions and Virtual Care (Lapointe-Shaw), Hôpital Women's College; ICES Central (Lapointe-Shaw, Rosella); Département de médecine (Weinerman), Centre des sciences de la santé Sunnybrook; Institute for Better Health (Tang, Rosella), Trillium Health Partners, Mississauga, Ont.; Département de génie mécanique et industriel (Chan), Université de Toronto; Département conjoint d'imagerie médicale (Cheung), Réseau universitaire de santé; Division d'épidémiologie (Cheung, Rosella), École de santé publique Dalla Lana; Institut Vecteur (Rosella, Ghassemi); Département d'informatique (Ghassemi) et Faculté de pharmacie Leslie Dan (Mamdani), Université de Toronto, Ont.

Collaborateurs : Tous les auteurs ont contribué à la conception de l'étude, et Amol Verma et Fahad Razak en ont élaboré la structure, avec une contribution substantielle de tous les auteurs. Amol Verma, Tejasvi Hora, Hae Young Jung, Michael Fralick, Sarah Malecki, Lauren Lapointe-Shaw, Adina Weinerman, Terence Tang, Janice Kwan, Jessica Liu, Shail Rawal et Fahad Razak ont contribué à la collecte de données. Tejasvi Hora et Hae Young Jung ont réalisé l'analyse des données. L'ensemble des auteurs ont collaboré à l'interprétation des données. Amol Verma a rédigé la première ébauche du manuscrit. Tous les auteurs ont révisé de façon critique le contenu intellectuel important du manuscrit; ils ont donné leur approbation finale pour la version destinée à être publiée et endossent l'entière responsabilité de tous les aspects du travail.

Propriété intellectuelle du contenu : Il s'agit d'un article en libre accès distribué conformément aux modalités de la licence Creative Commons Attribution (CC BY-NC-ND 4.0), qui permet l'utilisation, la diffusion et la reproduction de tout médium à la condition que la publication originale soit adéquatement citée, que l'utilisation se fasse à des fins non commerciales (c.-à-d., recherche ou éducation) et qu'aucune modification ni adaptation n'y soit apportée. Voir : https://creativecommons.org/licenses/by-nc-nd/4.0/deed.fr.

Soutien financier : Ce projet est soutenu par une subvention des IRSC VR4-172743. L'élaboration de la plateforme de données GEMINI a été rendue possible par le soutien financier de la Société canadienne du cancer, du Réseau canadien des soins aux personnes fragilisées, des Instituts de recherche en santé du Canada, de l'Association canadienne de protection médicale, de la fondation Green Shield Canada, du Conseil de recherches en sciences naturelles et en génie du Canada, de Santé Ontario, du Fonds pour l'innovation de l'association de l'hôpital St. Michael et du Département de médecine de l'Université de Toronto, ainsi que par des contributions en nature des hôpitaux partenaires et de l'Institut Vecteur. Les organismes subventionnaires n'ont exercé aucun rôle dans la conception, la réalisation ou l'interprétation des données de la présente étude.

Partage des données : Les données du présent manuscrit sont accessibles sur demande en communiquant avec l'auteur-ressource, dans la mesure où la demande est conforme aux exigences du comité d'éthique de la recherche local et des ententes de partage des données.

Remerciements : Les auteurs remercient la Dre Radha Koppula d'avoir réalisé l'extraction manuelle des tableaux et Daniel Tamming et Sudipta Saha pour leur contribution à l'analyse des données. Les auteurs remercient aussi toutes les personnes et tous les organismes qui ont fourni des données pour la présente étude.

Accepté : 28 janvier 2021

Correspondance : Amol Verma, amol.verma@mail.utoronto.ca 